\title{
Possible Beneficial Effect of Metformin Alone or in Combination with Methotrexate in Rheumatoid Arthritis Induced Rat Model
}

Nasr N. Makar, Abdel-Hamid M. Elhawary, Hanan T. Emam, Nashwa H. Abo Ria, Enas Shaaban

\begin{abstract}
Department of clinical pharmacology, Benha faculty of medicine, Benha University, Egypt

Correspondence to: Enas Shaaban, department of clinical pharmacology, Benha faculty of medicine, Benha University, Egypt
\end{abstract}

Email:

dr.enassokker@yahoo.com

Received: 31 July 2019

Accepted: 7 August 2019

\begin{abstract}
:
Background: Rheumatoid arthritis (RA) is a chronic, relapsing inflammatory and autoimmune multisystem illness that affects the joints. Methotrexate (MTX) is considered by many rheumatologists to be the most important and useful [DMARDs]. Metformin an antidiabetic medication has been reported to exert an anti-inflammatory effect. The rationale of this study is to test the effect of monotherapy with metformin alone or in comparison with methotrexate monotherapy on Adjuvant-induced arthritis (AIA). Aim of the study: The present study was designed to evaluate the effect of metformin alone and during basal treatment with methotrexate on rheumatoid factor, C-reactive protein, reduced glutathione, tumor necrosis factor- $\alpha$, arthritis score and histopathological changes. Materials and methods: Rats were classified into: Group I: control normal group. Group II: was not treated (diseased group). Group III was treated with methotrexate (MTX). Group IV: was treated with metformin. Group V: was treated with combination of MTX with metformin. All treated groups received drugs for 4 weeks. Results: All groups showed significant improvement in all parameters and improvement of the histopathology of the joint. A significant reduction in the
\end{abstract} score was seen in all treated group at the end of $3^{\text {rd }}$ and $4^{\text {th }}$ weeks. Conclusion: All tested drugs alone or in combination showed improvement of parameters of RA. It is found that all combinations show more efficacy than each drug alone.

Key words: Rheumatoid arthritis, methotrexate, metformin, adjuvant-induced arthritis 


\section{INTRODUCTION}

Rheumatoid arthritis (RA) is the most common rheumatologic disease [1]. It is characterized by a chronic inflammation of synovium, leading to progressive joint destruction. Although the cause of rheumatoid arthritis is unknown, autoimmunity plays an important role in both its chronicity and progression [2].

The pathogenesis of the rheumatoid joint involves hyperplasia of the synovial lining cells, mononuclear cell infiltration and new blood vessel formation within the synovium. Moreover, destruction of the cartilage and underlying bone occurs as a consequence of pro-inflammatory cytokines, particularly tumor necrosis factor- $\alpha(\mathrm{TNF}-\alpha)$ and proteases [3].

These cytokines can also reach systemic circulation and mediate inflammatory responses in other organs. Moreover, they stimulate phagocytic cells to produce reactive oxygen species (ROS), which mediate tissue injury in RA. Also, the accumulation of inflammatory cells was observed in synovitis of patients with active rheumatoid arthritis $[4, \mathbf{5}]$.

The current management of RA includes symptom-relieving nonsteroidal antiinflammatory drugs, disease-modifying antirheumatic drugs [DMARDs] such as azathioprine, methotrexate (MTX) [6]. Methotrexate (MTX) is considered by many rheumatologists to be the most important and useful [DMARDs] and is often part of the initial line of treatment. The suggested mechanisms of MTX include: (a) inhibition of T-cell proliferation by affecting purine and pyrimidine metabolism, (b) alter the recruitment of monocytes to the inflamed joint by interfering with glutathione metabolism [7, 8].

Methotrexate has shown to have toxic gastrointestinal, hematologic, pulmonary and hepatic adverse effects. Central nervous system reactions to methotrexate have been reported, which includes myelopathies and leuco-encephalopathies. It has a variety of cutaneous side effects, particularly when administered in high doses $[8,9]$.

The new trend of medical treatment of rheumatoid arthritis seeks for new drugs with more efficacy and less side effects since methotrexate causes many adverse effects and toxicities including pulmonary, hepatic, renal and cardiac [10]. So, there is a case for exploring newer therapies and regimes to be used as a monotherapy or as combinations with one of [DMARDs]. One strategy for 
treatment of RA is the design of drugs that can ameliorate inflammation [11].

Metformin was originally introduced as an antidiabetic medication. In addition to the glucose lowering effect, metformin has been reported to exert an antiinflammatory effect, which is also mediated by metformin-activated AMPK. Some experiments have shown the efficacy of metformin in collagen-induced arthritis (CIA) induced RA disease process [12].

These observations provided a rationale for testing the effect of monotherapy with metformin alone or in comparison with methotrexate monotherapy on Adjuvantinduced arthritis (AIA).

Also in this backdrop, we conducted the present study to explore if combination therapy of MTX with metformin offers some add-on benefit over monotherapy with MTX or metformine in male rats in AIA model.

\section{Materials and methods}

\section{A. Animals}

It is a prospective study carried out on 30 Adult male albino rats obtained from Experimental Animal Breeding Farm, Helwan-Cairo) weighing between 150$200 \mathrm{~g}$ (at the beginning of the study), were used for in-vivo experiments. They were acclimatized for one week and were caged (6 rat/ cage) in fully ventilated room at room temperature in the pharmacology department, Benha Faculty of Medicine. Rats were fed a standard chow with water. This study was approved from ethical committee of Benha Faculty of Medicine.

\section{B. Drugs and chemicals}

Complete Freund's adjuvant (CFA) (Sigma-Aldrich Chemical Company), Methotrexate (Minapharm., Egypt), Metformin (ADWIC., Egypt), Formalin, solution, neutral $10 \%$ formaline (El Gomhoria Pharmaceutical Chemical Co., ARE),Urethane , Ethyl carbamat, white crystals (Sigma Chemical Co., USA), Hematoxylin and eosin (E.Merk, Darmastadt.,)[Germany], Rheumatoid factor kits (CliniLab Company, Cairo, Egypt),CRP kits (eBioscience, UK), Tumor necrosis factor alpha (TNF- $\alpha$ ) kits ( USA \& Canada | R\&D Systems, Inc), Reduced Glutathione kits ( Biodiagnostic Co., Giza, Egypt)

\section{Induction of RA}

Complete Freund's Adjuvant Arthritis was induced by S.C injection of $0.4 \mathrm{ml}$ of compelete Freund's adjuvant in the right hind limb for 12 day in three doses (one dose every four days) [10]. 


\section{Experimental design:}

Rats were classified into 5 equal groups (6 rats in each group). Group I: Nonarthritic untreated healthy control group (Control normal group): This group received a standard chow and tap water with no medication. Group II: Untreated rats with adjuvant arthritis group: This group was injected with complete Freund's adjuvant to induce rheumatoid arthritis. Group III: Rheumatoid arthritis (RA) rats, methotrexate treated group: This group was treated with methotrexate at a dose (0.6 mg/kg/week/by gavage) [13] for 4 weeks after induction of rheumatoid arthritis. Group IV: Rheumatoid arthritis (RA) rats, metformin treated group: This group was treated with metformin at a dose $(30 \mathrm{mg} / \mathrm{kg} /$ day $/$ by gavage) [14] for 4 weeks after induction of rheumatoid arthritis. Group V: Rheumatoid arthritis (RA) rats, (methotrexate and metformin) treated group: This group was treated with combination of methotrexate $(0.6$ $\mathrm{mg} / \mathrm{kg} /$ week/by gavage) and metformin (30 $\mathrm{mg} / \mathrm{kg} /$ day/by gavage) for 4 weeks after induction of rheumatoid arthritis. All treated groups received drugs for 4 weeks. Dose selection was based on previously published studies and pilot experiments.
At the end of experiment a blood sample of about $2 \mathrm{ml}$ was withdrawn by unheparinized cannula from right carotid artery [15]. The blood samples (each=2ml) were allowed to clot at room temperature, centrifuged at 3000 rotation/minute and the sera were separated. Samples were stored at $-20 \quad \dot{C}$ in dark containers for measurement of rheumatoid factor (RF), C-reactive protein (CRP), tumor necrosis factor alpha (TNF- $\alpha)$ and reduced glutathione (GSH).

Rats were euthanized at the end of the study and hind paw joints were removed and weighed. They were embedded in paraffin after fixing in formalin solution (10\% neutral buffered). Sections were cut in various slices having thickness of $6 \square \mathrm{m}$ and examined under microscope after staining with hematoxylin-eosin for perivascular inflammatory cell infiltrate in synovium and morphological changes including synovial cell hyperplasia and proliferation and villous hyperplasia [16].

\section{E.Assessment of arthritis}

a- Rats were scored for arthritis (arthritis index) by a set visual criterion at the end of each week of experiment according to the following criteria: No change $=0$, Erythema $=1$, Mild swelling $=2$, Gross swelling $=3$, Gross swelling and deformity $=4[17]$. 
b- Microscopic examination of sections of paw joint stained by $H \& E$ stain.

\section{F.Biochemical assays}

a- Serum RF was determined using an immunoenzymatic assay [18].

b- Serum TNF- $\alpha$ was measured by ELISA [19].

c- Serum CRP was determined using an enzyme-linked immunosorbent assay [20].

d- Serum GSH was determined by using a colorimetric method [21].

\section{Statistical analysis:}

Data were tabulated, coded then analyzed using the computer program SPSS (Statistical package for social science) version 23.0 to obtain descriptive data. Descriptive statistics were calculated in the form of Mean \pm Standard deviation (SD).

In the statistical comparison between the different groups, the significance of difference was tested using one way ANOVA (analysis of variance) to compare between more than two groups of numerical (parametric) data followed by post-hoc tukey. A $\mathrm{P}$ value $<0.05$ was considered statistically significant.

\section{Results:}

S.C injection of (CFA) resulted in significant increase in level of RF, CRP, TNF- $\alpha$ compared to control normal group.
Also, there is significant increase in arthritic score in diseased group every week without treatment compared to the score at $1^{\text {st }}$ day before adjuvant injection. While there was decrease in GSH level compared to control normal group. Joints obtained from AIA group showed proliferated blood vessels, prominent inflammatory infiltrate and villous formation.

Regarding, monotherapy treated groups leaded to significant improvement in serum RF, TNF- $\alpha$, CRP, GSH compared to control and diseased groups. Monotherapy with methotrexate showed the best results.

In addition, combination between MTX and metformin leaded to significant improvement in serum RF, TNF- $\alpha$, CRP, GSH compared to control and diseased groups. They showed better result than monotherapy treated groups.

Regarding arthritis score, a significant reduction in the score was seen in all treated group at the end of 3rd and 4th weeks compared to diseased group.

Histopathology indicates improvement of the joint as regard synovial hyperplasia, cartilage degeneration and inflammatory cell infilteration. This improvement was observed in all treated groups with best result in combination group. 
Table (1): The effect of treatment with metformin alone or in combination with methotrexate on (serum RF, TNF- $\alpha$, CRP, GSH), on experimentally induced rheumatoid arthritis, in rats:

\begin{tabular}{|c|c|c|c|c|}
\hline Group-parameter & RF (IU/ml) & TNF- $\alpha(\mathrm{pg} / \mathrm{ml})$ & $\operatorname{CRP}(\mathrm{mg} / \mathrm{dl})$ & GSH(mg/dl) \\
\hline Normal control group & $12.28 \pm 2.456$ & $12.22 \pm 2.444$ & $4.1 \pm 0.42$ & $23.75 \pm 2.39$ \\
\hline $\begin{array}{l}\text { Arthritic group (Diseased } \\
\text { group) }\end{array}$ & $64.4 \pm 4.1 *$ & $87.82 \pm 14.64 *$ & $14.82 \pm 1.43^{*}$ & $4.1 \pm 1.43 *$ \\
\hline MTX treated group & $29.77 \pm 4.154 * \#$ & $31.63 \pm 3.326^{* \#}$ & $8.19 \pm 0.63 * \#$ & $15.97 \pm 1.69 * \#$ \\
\hline Met treated group & $48.34 \pm 4.068 * \# \$$ & $57.8 \pm 5.16 * \# \$$ & $11.33 \pm 0.64 * \# \$$ & $9.48 \pm 1.1 * \# \$$ \\
\hline MTX + Met treated group & $17.93 \pm 3.986 \# \$ \alpha$ & $16.17 \pm 4.234 \# \$ \alpha$ & $5.12 \pm 0.73 \# \$ \alpha$ & $19.55 \pm 1.36 * \# \$ \alpha$ \\
\hline
\end{tabular}

*: Significant difference versus control group at $\mathrm{p}<0.05$

\#: Significant difference versus diseased group at $p<0.05$

\$: Significant difference versus MTX treated group at $\mathrm{p}<0.05$

$\alpha$ : Significant difference versus Met treated group at $\mathrm{p}<0.05$

Table (2): The effect of induction of adjuvant arthritis by $(0.4 \mathrm{ml}$ complete Frenud's adjuvant $)$ in rats, on arthritic score, at different times:

\begin{tabular}{lllllll}
\hline & $\begin{array}{l}\mathbf{1}^{\text {st }} \text { day } \\
\text { before } \\
\text { CFA } \\
\text { injection }\end{array}$ & $\begin{array}{l}\text { End of } 3 \\
\text { doses of } \\
\text { CFA }\end{array}$ & $\begin{array}{l}\text { End of } 1^{\text {st }} \\
\text { Week } \\
\text { TTT }\end{array}$ & $\begin{array}{l}\text { End of 2nd } \\
\text { week of } \\
\text { TTT }\end{array}$ & $\begin{array}{l}\text { End of 3rd } \\
\text { week of } \\
\text { TTT }\end{array}$ & $\begin{array}{l}\text { End of 4th } \\
\text { week of } \\
\text { TTT }\end{array}$ \\
\hline $\begin{array}{l}\text { Diseased } \\
\text { group }\end{array}$ & $0.0 \pm 0.0$ & $2.3 \pm 0.46 \#$ & $3.5 \pm 0.8 *$ & $3.5 \pm 0.6^{*}$ & $4 \pm 0.67 *$ & $4 \pm 0.57 *$ \\
\hline
\end{tabular}

\#: Significant difference versus diseased group at 1st day before CFA injection at $p<0.05$

*: Significant difference versus diseased group at the end of 3 doses of CFA at $p<0.05$

Table (3): The effect of treatment with metformin alone or in combination with methotrexate, on arthritic score at different times, on experimentally induced rheumatoid arthritis in rats:

\begin{tabular}{cllll}
\hline & $\begin{array}{l}\text { Diseased } \\
\text { group }\end{array}$ & $\begin{array}{l}\text { MTX } \\
\text { treated } \\
\text { group }\end{array}$ & $\begin{array}{l}\text { Met treated } \\
\text { group }\end{array}$ & $\begin{array}{l}\text { MTX+Met } \\
\text { treated } \\
\text { group }\end{array}$ \\
\hline $\begin{array}{c}\text { End of 3 dose of } \\
\text { CFA }\end{array}$ & $2.3 \pm 0.46$ & $2 \pm 0.36$ & $2 \pm 0.56$ & $1.8 \pm 0.38$ \\
End of 1st w TTT & $3.5 \pm 0.8$ & $2.9 \pm 0.5$ & $3 \pm 0.5$ & $2.7 \pm 0.68$ \\
End of 2nd w TTT & $3.5 \pm 0.6$ & $2.62 \pm 0.6$ & $2.8 \pm 0.6$ & $2.26 \pm 0.4$ \\
End of 3rd w TTT & $4 \pm 0.67$ & $2.31 \pm 0.4 \#$ & $2.77 \pm 0.5 \#$ & $1.57 \pm 0.18 \# \$ \infty$ \\
End of 4th w TTT & $4 \pm 0.57$ & $1.8 \pm 0.17 \#$ & $2.67 \pm 0.14 \# \$$ & $1.11 \pm 0.18 \# \$ \infty$ \\
\hline
\end{tabular}

\#: Significant difference versus diseased group at $\mathrm{p}<0.05$

$\$$ : Significant difference versus MTX treated group at $\mathrm{p}<0.05$

$\infty$ : Significant difference versus Met treated group at $p<0.05$ 


\section{Histopathological changes:}

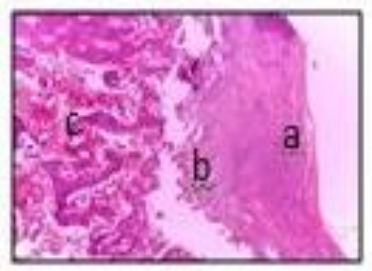

(1)

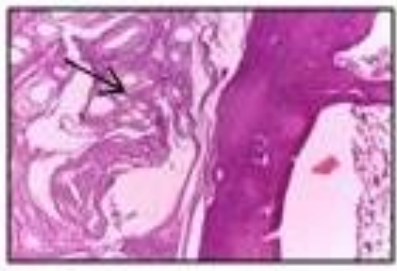

(2)

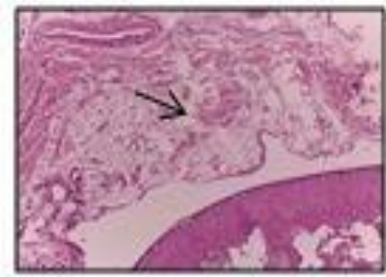

(3)

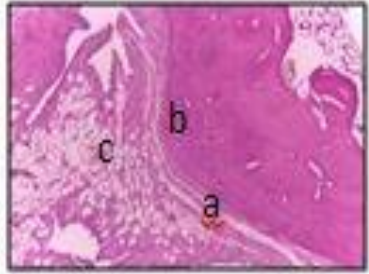

(4)

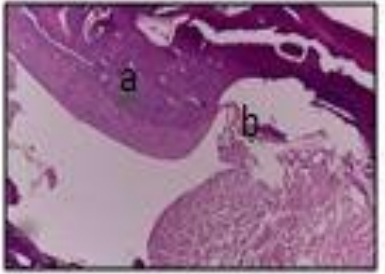

(5)

figure (1): Photomicrograph of a cut section in normal rat joint treated with galantamine shows (a) flattened superficial layer, (b) homogenous matrix and associated with (c) bone trabeculae and bone marrow (H\&E x100)

figure (2): Photomicrogragh of a cut section in rat joint of AA group shows inflammatory infiltrate and proliferated blood vessels (black arrow) (H\&E x 100).

figure (3): Photomicrogragh of a cut section in rat joint of MTX treated group shows improvement of in inflammatory response (black arrow) (H\&E x100).

figure (4): Photomicrogragh of a cut section in rat joint of metformin treated group shows (a) flattening of superficial layer of cartilage associated with (b) large compact layer (c) improvement of inflammatory infiltrate (H\&E x100).

figure (5): Photomicrogragh of a cut section in rat joint of (methotrexate and metformin) treated group shows flattening of superficial layer (b) improvement of inflammatory infiltrate and compact layer (H\&E x 100).

\section{Discussion:}

The choice of CFA in this study because it has more similar disease features with human RA than CIA. Rodent adjuvant arthritis, as an experimental model, resembles RA in histological pathology, pannus formation and a number of angiogenic mediators, including cytokines and growth factors. The similarities in joint pathology between AA and RA could be exerted for screening of new drugs for treatment of RA disease. The male wistar rats have been found to be more susceptible to RA, particularly in terms of onset and severity of the disease process $[\mathbf{2 2}, \mathbf{2 3}]$.

The data of the present work revealed that s.c. injection of (CFA) resulted in increase in level of RF, CRP, TNF- $\alpha$, arthritic score, decrease in level of GSH and affected the pathology of the joint. This finding is similar to the observations of Refaat et alwho proved 
that injection of CFA led to significant elevation of serum RF, serum TNF- $\alpha$ and decrease blood GSH level compared to normal control group [24]. Also, these results are in agreement with the study of Patel and Pundarikakshudu who reported that injection of complete Freund's adjuvant leads to edematous inflammation, increased vascularity owing to vasodilation, marked inflammatory cell infiltration compared to normal control group[25] .

Activation of inflammatory mediators TNF- $\alpha$, IL- $1 \beta$, and IL- 6 by CFA aggravates the oxidative damage of the vital organs in rheumatoid arthritis, such as the liver. The liver, in turn, influences the systemic inflammation via producing inflammatory cytokines and mediators such as TNF- $\alpha$, IL$1 \beta$, IL-6 and NO. IL-6, IL-1 $\beta$, and TNF- $\alpha$ promote the synthesis of CRP in hepatocytes

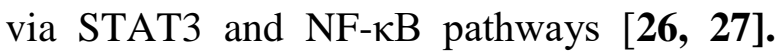
Also, the pro-inflammatory cytokines TNF- $\alpha$, IL-1, and IL-2 have been shown to play an important role in the pathophysiology of arthritis development in animal models and humans. It was reported that increased expression of inflammatory cytokines, including TNF- $\alpha$ and IL-1 $\beta$, was observed in the bone region of the joint or serum samples from human osteoarthritis or rheumatoid arthritic patients [28].
Methotrexate significantly improved all tested parameters of rheumatoid arthritis, arthritic score and histopathology of the joints. The result of this study is in agreement with the observations of Kaneko et al. who reported that administration of MTX as a treatment after induction of RA by CFA, induced a highly significant decrease in serum RF, serum TNF- $\alpha$, serum CRP and induced a highly significant increase in blood GSH level compared with arthritic rats[29]. Also, Wang study is in line with our results as MTX administration decreased the arthritis score significantly [30]. In addition, our results are in agreement with the study which reported that MTX showed moderate improvement in the form of decrease in villous formation [31]. In Contrast to the previous results, another study reported that the levels of GSH with MTX were markedly suppressed in the arthritic group compared to the normal one[32] .

Some researchers have postulated that MTX reduces plasma TNF- $\alpha$ level by suppressing transcriptional activity rather than by suppressing lymphocyte proliferation. Also, it has role in inhibition of trans-methylation reaction necessary for T-cell cytotoxicity prevention and alter the recruitment of monocytes to the inflamed joint by interfering with glutathione metabolism alterations in recruitment of monocytes and other cells to the inflamed joint, and promotion of the 
release of the endogenous anti-inflammatory mediator adenosine $[\mathbf{7}, 33]$. Improvement of CRP by methotrexate can be explained by its anti-inflammatory role in the form of improvement of tumor necrosis factor alpha. As IL-6, IL-1 $\beta$, and TNF- $\alpha$ promote the synthesis of CRP in hepatocytes via STAT3 and NF-kB pathways [26, 27].

MTX treatment caused significant improvement of the arthritis index as well as modulation of the altered parameters produced in arthritic rats with respect to antioxidants levels .Also, this can be attributed to its established anti-inflammatory, antiproliferative, immunosuppressive effects on activated $\mathrm{T}$ lymphocytes, increasing the rate of apoptosis of $\mathrm{T}$ cells, increasing endogenous adenosine release, altering the expression of cellular adhesion molecules, influencing production of cytokines, humoral responses and bone formation[34].

Metformin significantly improved all markers of rheumatoid arthritis, arthritis score and histopathology of the joints, but it was less effective than methotrexate. Hyun et al. study supported these results, as his study resulted in significant decrease of TNF$\alpha$ production in mice fed by high fat diet by metformin [35]. Also, these data are in harmony with a study which showed the effect of metformin in reduction of CRP diabetic rats [36]. In addition, later on 2016 a study reported that GSH concentration was improved in diabetic treated rats by metformin [37]. Consistent with the arthritis score with metformin, Kim et al. study showed minimal signs of inflammation were detected in the metformin-treated obese CIA mice [38].

Metformin suppresses the production of TNF- $\alpha$ and attenuates gene and protein expression of TNF- $\alpha$ in macrophages and it regulates inflammation through downregulation of scavenger receptors in macrophages [35].

As infiltrated macrophages in inflamed area, attract other macrophages and accumulated macrophages secrete pro-inflammatory cytokines such as IL-1 $\beta$, IL-6 and tumor necrosis factor (TNF)- $\alpha$. Pro-inflammatory cytokines promote unnecessary inflammation [39].

Numerous studies have subsequently reported that scavenger receptors, which are expressed by macrophage, can recognize and clear modified host component, apoptotic cells and pathogens, indicating that scavenger receptors play an essential role in innate immunity and inflammatory responses [40].Scavenger receptors enhance NF- $\mathrm{B}$ activity, leading to pro-inflammatory cytokine production [41]. 
The improvement in the mitochondrial function and energy production induced by metformin may reduce the production of the inflammatory mediators such as CRP, TNF$\alpha$ and IL-6 [42].

Several studies have shown that the primary effect of metformin as antioxidant is the inhibition of mitochondrial complex I (NADH: ubiquinone oxidoreductase) [43]. Mitochondrial complex I may contribute substantially to the cellular ROS production [44].

It is well documented that a blockage this complex leads to decreased production of reactive species, due to reduced transport of electrons from NADH plus $\mathrm{H}^{+}$(45). Therefore, evidence suggests that metformin reduces endogenous ROS mitochondrial levels [46].

Concomitant administration of metformin with MTX produced better results in all parameters of rheumatoid arthritis, arthritic score and histopathology of the joints, than each drug alone. This finding is in agreement with the observations of Hisadome et al. study who reported that antiTNF- $\alpha$ in combination with MTX markedly decreases joint destruction in RA and synergistically suppressed arthritic progression [47].

\section{Conclusion:}

Metformin alone or in combination with methotrexate improves adjuvant arthritis. When a comparison between combinations and each drug alone is carried out, it is found that all combinations showed more efficacy than each drug alone. Metformin can be used alone or in combination with MTX treatment of rheumatoid arthritis to avoid possible side effect of high doses of methotrexate

\section{References:}

(1) Lai, C. H.; Lai, M. S.; Lai, K. L. et al., (2012): Nationwide population-based epidemiologic study of rheumatoid arthritis in Taiwan. Clin Exp Rheumatol, 30:358-363.

(2) Hendawy, O. M.; Parambi D. G. (2018): Effect of Boswellia on Adjuvant Induced Rheumatoid Arthritis in Experimental Animals. International Journal of Phytomedicine, 10(2):94.

(3)Feldmann, M.; Brennan, F. M.; Foxwell, B. M. et al.,(2005): Anti-TNF therapy: Where have we got to in 2005? J. Autoimmun., 25: 26-28.

(4) Hsu, H. C.; Wu, Y.; Mountz, J. D. (2006): Tumor necrosis factor ligand-receptor superfamily and arthritis. Current directions in autoimmunity, 9:37-54

(5) Comar, J. F.; Babeto De Sá-Nakanishi, A.; De Oliveira A. L. et al., (2013): Oxidative state of the liver of rats with adjuvant-induced arthritis. Free Radical Biology \& Medicine, 58:144-153.

(6) Gibofsky, A. (2006): Combination therapy for rheumatoid arthritis in the era of biologicals. HSS J, 2:30-41.

(7) Cronstein, B. N. (2005): Low-dose methotrexate: A mainstay in the treatment of rheumatoid arthritis. Pharmacol Rev, 57:163-72.

(8)Wasserman, B. R.; Moskovich, R.; Razi, A. E. (2011): Rheumatoid arthritis of the cervical spine-clinical considerations. Bulletin of the New York University Hospital for Joint Diseases, 69(2):136148 . 
(9) Scheinfeld, N. (2006): Three cases of toxic skin eruptions associated with methotrexate and a compilation of methotrexate-induced skin eruptions. Dermatology online journal, 12 (7):15.

(10) Hendawy, O. M.; Ahmed, W. M. S.; Abosaif, A. A. et al., (2015): Effect of atorvastatin and vitamin $\mathrm{D}$ on freund adjuvant-induced rheumatoid arthritis in rat . J Bioequiv Availab., 7:090-094. doi: 10.4172/jbb.100221.

(11) Roy, T.; Banerjee, I.; Ghosh, S. et al., (2017): Effects of co-treatment with pioglitazone and methotrexate on experimentally induced rheumatoid arthritis in Wistar albino rats. Indian J Pharmacol., 49:168-75.

(12) Son, H.; Lee, J.; Lee, S. (2014): Metformin Attenuates Experimental Autoimmune Arthritis through Reciprocal Regulation of Th17/Treg Balance and Osteoclastogenesis. Mediators Inflamm. , 2014: 973986.

(13) Rovenský, J.; Švík, K.; Stanciková, M. et al., (2006): The effects of selenium enriched enterococus faecium M-74 on methotrexate treatment of rats with adjuvant arthritis. International Journal of Probiotics and Prebiotics, 1(2):137-144.

(14)Wessels, B.; Ciapaite, J.;van den Broek, N. M.A. et al., (2014): Metformin Impairs Mitochondrial Function in Skeletal Muscle of Both Lean and Diabetic Rats in a Dose-Dependent Manner journal.pone. June 20, doi.org/10.1371/.0100525

(15)Yamamoto, K.; Masuyama, T.; Sakata, Y.; Mano, T.; Nishikawa, N. et al., (2000): Roles of reninangiotensin and endothelin systems in development of diastolic heart failure in hypertensive hearts.Cardiovascular research, 47:274-283.

(16)Faisal, R.; Ahmad, N.; Fahad, Y. S. et al.,(2018): Anti-arthritic effect of thymoquinonein comparison with methotrexate on pristine induced arthritis in female Sprague dawely rats. J Ayub Med Coll Abbottabad.,30(1)

(17) Sachin, V.;Subhash, B. L.;Vishwaraman, M. et al., (2013): Anti-inflammatory and antiarthritic activity of type -A procyanidine polyphenols from bark of Cinnamomumzeylanicum in rats. Food Sci Hum Wellness, 2:5967.

(18) Cook, L.; Angello, V.; Rose, R .N. et al., (1992): Rheumatoid factor ELISA Kit. Manual of clinical lab immunology. TX, USA: 1992. 762.

(19) Kasumagic-Halilovic, E.; Prohic, A.; Cavaljuga, S. (2011): Tumor necrosis factor-alpha in patients with alopecia areata. Indian Journal of Dermatology, 56(5):494-6

(20)Nathan, B. R.; Scheld, W. M. (2002): The potential roles of C-reactive protein and procalcitonin concentrations in the serum and cerebrospinal fluid in the diagnosis of bacterial meningitis. Curr Clin Top Infect Dis., 22 :155-165.

(21) Beutler, E.; Duron, O.; Kelly, M. B. (1963): Glutathione reduced. J Lab Clin Med.; 61 :882.

(22)Lorenzo,N.; Domínguez,M.C.; Hernández,A.etal., A. (2008): Characterization of two experimental rodent models for evaluating novel drugs for rheumatoid arthritis.Biotecnología Aplicada, 25, 236-241.

(23)Snekhalatha, U.; Anburajan, M.; Venkatraman, B. et al., (2013): Evaluation of complete Freund's adjuvant-induced arthritis in a Wistar rat model. Comparison of thermography and histopathology. Z Rheumatol.,72:375-82.

(24)Refaat, R.; Salama, M.; Abdel Meguid, E. et al., (2013): Evaluation of the effect of losartan and methotrexate combined therapy in adjuvant-induced arthritis in rats. Eur. J. Pharmacol., 698: 421-428.

(25) Patel, M. G.; Pundarikakshudu' K. (2016): Antiarthritic activity of a classical Ayurvedic formulation Vatari Guggulu in rats. $\mathbf{J}$ Tradit Complement Med., 6(4): 389-394.

(26) Zhang, D.; Sun, M.; Samols, D.; Kushner, I. (1996): STAT3 participates in transcriptional activation of the C-reactive protein gene by interleukin-6. J Biol Chem., 271(16):9503-9.

(27) Kleemann, R.; Gervois, P. P.; Verschuren, L. (2003): Fibrates down-regulate IL-1-stimulated Creactive protein gene expression in hepatocytes by reducing nuclear p50-NFkappa B-C/EBP-beta complex formation. Blood, 101(2):545-51.

(28)Kaneko, M.; Tomita, T.; Nakase, T. et al., (2001): Expression of proteinases and inflammatory cytokines in subchondral bone regions in the destructive joint of rheumatoid arthritis Rheumatology (Oxford), 40(3):247-55.

(29)Mohamed, M. A.; Mahmoud, M. F.; Rezk, A. M. (2014): Effect of pentoxifylline and pioglitazone on rheumatoid arthritis induced experimentally in rats. Menoufia Med J, 27(4):766-774.

(30)Wang, X.; Yan, X.; Wang, F. et al., (2018): Role of methotrexate chronotherapy in collagen-induced rheumatoid arthritis in rats. Z Rheumatol., 77(3):249-255. 
(31)Mohammad, M. O.; Agha, A.M.;, Abdul-Gawad, H. M. et al., (2003): Effects of methotrexate and leflunamide therapy on knee synoviumof adjuvant induced arthritis as a model for rheumatoid arthritis. Egypt Rheumatol Rehab., 30, (1).

(32)Ismail, M.; Hasan, H.; El-Orfali, Y. et al., (2018): Anti-Inflammatory, Antioxidative, and Hepatoprotective Effects of Trans 9Tetrahydrocannabinol/Sesame Oil on AdjuvantInduced Arthritis in Rats Evidence-Based Complementary and Alternative Medicine Volume 2018, Article ID 9365464, 13 pages.

(33)To, H.; Yoshimatsu, H.; Tomonari, M.; et al., (2011): Methotrexate chronotherapy is effective against rheumatoid arthritis. Chronobiol Int., 28:267-274.

(34) Shahin, D.; Eltoraby, E.; Mesbah, A. et al., (2010): Insulin resistance in early untreated rheumatoid arthritis patients. Clin Biochem., 43(78):661-5.

(35)Hyun, B.; Shin, S.; Lee, A. et al., (2013): Metformin Down-regulates TNF- $\alpha$ Secretion via Suppression of Scavenger Receptors in Macrophages. Immune Network, 13(4):123-132.

(36) Salman, Z. K.; Refaat, R.; Selima, E. et al., (2013): The combined effect of metformin and Lcysteine on inflammation, oxidative stress and insulin resistance in streptozotocin-induced type 2 diabetes in rats. Eur J Pharmacol., 714 (1-3):448-55.

(37) Obi, B. C.; Okoye, T. C.; Okpashi, V. E. et al., (2016): Comparative Study of the Antioxidant Effects of Metformin, Glibenclamide, and Repaglinide in Alloxan-Induced Diabetic Rats. J Diabetes Res., 2016:1635361.

(38) Kim, E.; Lee, s.; Lee, s. et al., (2018): Metformin ameliorates experimental-obesityassociated autoimmune arthritis by inducing FGF21 expression and brown adipocyte differentiation. Experimental \& Molecular Medicine, 50: 432 .
(39)Patel, P. S.; Buras, E. D.; Balasubramanyam, A. (2013): The role of the immune system in obesity and insulin resistance. J Obes., 2013:616193.

(40)Kzhyshkowska, J.; Neyen, C.; Gordon, S. (2012): Role of macrophage scavenger receptors in atherosclerosis. Immunobiology. 217(5):492-502.

(41)Yu, H.; Ha, T.; Liu, L.; et al., (2012): Scavenger receptor A (SR-A) is required for LPS-induced TLR4 mediated NF- $\kappa \mathrm{B}$ activation in macrophages. Biochim Biophys Acta., 1823(7):1192-8.

(42)Alhaider, A. A.; Korashy, H. M.; Sayed-Ahmed, M. M. et al., (2011): Metformin attenuates streptozotocin induced diabetic nephropathy in rats through modulation of oxidative stress genes expression. Chemico-Biological interactions, 192:233-242.

(43)Carvalho, C.; Correia, S.; Santos, M. S. et al., (2008): Metformin promotes isolated rat liver mitochondria impairment. Molecular and Cellular Biochemistry, 308(1-2):75-83.

(44)Vinothkumar, K. R.; Zhu, J.; Hirst, J. (2014): "Architecture of mammalian respiratory complex I. Nature, 515(7525):80-84.

(45)Kushnareva, Y.; Murphy, A. N.; Andreyev, A. (2002): Complex I-mediated reactive oxygen species generation: modulation by cytochrome $\mathrm{c}$ and $\mathrm{NAD}(\mathrm{P})+$ oxidation-reduction state. Biochemical Journal, 368(2):545-553.

(46)Kelly, B.; Tannahill, G. M.; Murphy, M. P.; L. O'Neill, A. J. (2015): Metformin inhibits the production of reactive oxygen species from NADH:ubiquinone oxidoreductase to limit induction of interleukin-1 $\beta$ (IL-1 $\beta)$ and boosts interleukin-10 (IL-10) in lipopolysaccharide (LPS)-activated macrophages. The Journal of Biological Chemistry, 290(33):20348-20359.

(47) Hisadome, M.; Fukuda, T.; Adachi, K. et al.,(2004): Combination benefit of a pyrimidylpiperazine derivative (Y-40138) and methotrexate in arthritic rats. European Journal of Pharmacology,497(3): 351-359.

To cite this article: Nasr N. Makar , Abdel-Hamid M. Elhawary, Hanan T. Emam, Nashwa H. Abo Ria, Enas Shaaban Possible Beneficial Effect of Metformin Alone or in Combination with Methotrexate in Rheumatoid Arthritis Induced Rat Model, BMFJ 2019; 37(1):143-154. DOI: $10.21608 / \mathrm{bmfj} .2020 .15502 .1030$ 